\title{
A Theorem on Ergodicity of Two-Dimensional Hyperbolic Billiards
}

\section{A. Bunimovich ${ }^{\star}$}

Shirshov Institute of Oceanology of the Academy of Sciences, USSR, SU-117218 Moscow, USSR

\begin{abstract}
Ergodicity of two-dimensional billiards which satisfy some general conditions is proved. This theorem is applied to one concrete class of billiards that contains, in particular, billiards in the "stadium."
\end{abstract}

Dynamic systems of the billiard type (or simply billiards) belong to one of the most popular and best investigated class of hyperbolic dynamical systems with singularities. Hyperbolicity leads to entailing strong stochastic properties of the system such as positivity of Kolmogorov-Sinai entropy, existence of ergodic components of positive measure etc. Nevertheless the usual proof (attributed to Hopf $[\mathrm{H}]$ ) of the ergodicity of smooth hyperbolic dynamic systems (for instance Anosov systems) cannot be applied to billiards in view of the presence of singularities. Sinai pointed out first (and proved it in [S1] for Sinai billiards on $\mathbb{T}^{2}$ ) a new statement that gives the possibility of applying Hopf's idea for the proof of ergodicity of hyperbolic dynamic systems with singularities. In [BS] the proof of this statement was simplified and extended to a wider class of Sinai billiards. The corresponding assertion was named in [BS] the main theorem of the theory of Sinai billiards. Later [B1] the same assertion was used for the proof of ergodicity of billiards in domains with focusing components of the boundary. Therefore it is now called the main theorem of the theory of billards with hyperbolic behavior.

Sinai proposed in $[\mathrm{S} 2, \mathrm{~S} 3]$ a more general method for the proof of this theorem. Making use of this method it was possible in [SC] to prove the ergodicity of some classes of semidispersing billiards. Especially one has to mention the proof of ergodicity of the system of three billiard balls on the $d$-dimensional torus [KSS1] which was recently obtained by Kramli, Simanyi, Szász [KSS2]. The authors of [KSS1] used a modified version of the main theorem which they called a "transversal" fundamental theorem for semi-dispersing billiards [KSS2]. Here

* This paper was completed during the author's stay at Rutgers University where he was supported by NSF Grant DHS 89-01545 and at Courant Institute of Mathematical Sciences where he was supported by a Grant from the Alfred P. Sloan Foundation 
we use the method of [S2, S3, CS, KSS2] (which we call below the new one in order to distinguish it from the old method of [S1, BS]) to prove the main theorem for a general class of hyperbolic billiards which includes in particular billiards in domains having boundaries with focusing components. The scheme of our proof is exactly the same as in [SC, KSS2].

The structure of the paper is the following. In Sect. 1 we formulate axiomatically the properties of some abstract class of billiards, for which the main theorem is proven in Sect. 2. In Sect. 3 we verify the conditions of this theorem for a class of billiards in domains with focusing components of the boundary. Section 4 contains some comments on the interrelation between the old and new methods of the proof of the main theorem. We omit proofs of those statements which completely coincide with the corresponding proofs in [SC, $\mathrm{KSS} 2]$ and give detailed references.

\section{Definition and Main Properties of Billiards Under Consideration}

Let $Q$ be a bounded closed domain in the Euclidean plane $\mathbb{R}^{2}$ or on the twodimensional torus $\mathbb{T}^{2}$ with Euclidean metric. We assume that the boundary $\partial Q$ consists of a finite number of smooth (of class $C^{3}$ ) nonselfintersecting curves $\partial Q_{i}$, $i=1, \ldots, p$, which we shall call regular components of $\partial Q$. Hence every regular component of the boundary is closed or has common endpoints with other regular components of $\partial Q$. The points of intersection of different regular components of the boundary will be called singular points. $\partial Q$ has at most a finite number of singular points. We denote these points $\hat{q}_{1}, \ldots, \hat{q}_{L}$ and let $\bigcup_{i=1}^{L} \hat{q}_{i}=V$.

We assume that the boundary $\partial Q$ is equipped with the field of unit inner normal vectors $n(q), q \in \partial Q$. We shall assume that the curvature of every regular component $\partial Q_{i}, i=1, \ldots, p$, of the boundary has the same sign at all points or is equal to zero. We denote by $\partial Q^{+}, \partial Q^{-}$, and $\partial Q^{0}$ the union of all regular components with positive, negative and zero curvature, respectively. Regular components of the boundary belonging to $\partial Q^{+}, \partial Q^{-}$, and $\partial Q^{0}$ will be called dispersing, focusing and neutral components, respectively. Besides we assume that $Q$ is not a polygon, i.e. $\partial Q^{+} \cup \partial Q^{-} \neq \emptyset$, and dispersing components intersect each other and neutral components of $\partial Q$ transversally.

Furthermore we denote by $M$ the restriction to $Q$ of the unit tangent bundle of $\mathbb{R}^{2}\left(\mathbb{T}^{2}\right)$. The preimage $\pi^{-1}(q)=S^{1}(q), q \in Q$, consists of unit vectors that are tangent to $Q$ at the point $q \in Q$. Points $x=(q, v) \in M$ will be called unit tangent vectors and $q=\pi(x)$ is the footpoint of $x$. It is easy to see that $M$ is the threedimensional manifold with the boundary $\partial M=\bigcup_{i=1}^{P} \pi^{-1}\left(\partial Q_{i}\right)=\bigcup_{i=1}^{p} \partial M^{(i)}$. In every regular component $\partial M^{(i)}$ we introduce a coordinate system $(r, \phi)$, where $r$ is the normalized arc length along $\partial Q_{i}$ and $\phi,-\pi<\phi \leqq \pi$, is the angle between the line element $x$ and the vector $n(q)$, where $q=\pi(x) \in \partial Q$.

A billiard in the domain $Q$ is the dynamical system generated by the motion of line elements $x \in M$ along geodesics with unit speed and with reflection from the boundary according to the law "angle of incidence equals angle of reflection." This means that if the geodesics intersect the boundary at a regular point along the 
direction $v$, the further motion occurs in the direction of the vector $v^{\prime}=v$ $-2(v, n(q)) n(q)$, where $q$ is the point where the intersection occurs and $(v, n(q))$ is the scalar product of the vectors $v$ and $n(q)$.

If a trajectory reaches a singular point then the further motion is not defined.

Let $M_{1}=\{x \in \partial M:(x, n(q)) \geqq 0, q=\pi(x)\}, M_{1}^{(i)}=M_{1} \cap \partial M^{(i)}$. It is clear that $M_{1}$ in coordinates $(r, \phi)$, is the union of not more than $p$ cylinders. We put $M_{1}^{ \pm}=M_{1}$ $\cap \pi^{-1}\left(\partial Q^{ \pm}\right), M_{1}^{0}=M_{1} \cap \pi^{-1}\left(\partial Q^{0}\right)$.

We denote $S_{0}=\{x \in \partial M:(x, n(q))=0\}, V_{0}=\pi^{-1}(V)$. The set $R_{0}=S_{0} \cup V_{0}$ will be called the set of singular points of the boundary.

We introduce in $M$ the measure $d \mu=$ const $d q d \omega$, where $d q$ is the measure in $Q$ induced by the Euclidean metric, $d \omega$ is the uniform measure on the sphere $S^{1}(q)$ $=\pi^{-1}(q)$ and const is a normalizing factor. The one-parameter group $\left\{T^{t}\right\}$ is a flow in the sense of ergodic theory.

For any point $x \in M$ we denote by $\tau^{+}(x)\left(\tau^{-}(x)\right)$ the nearest positive (negative) moment of reflection of the trajectory of $x$ from $\partial Q$. It is easy to see that $\tau^{+}(x)$ $<\infty\left(\left|\tau^{-}(x)\right|<\infty\right)$ for $\mu$ almost all points $x \in M$. Denote $\pi_{1}^{+} x=T^{\tau^{+}(x)+0} x, \pi_{1}^{-} x$ $=T^{\tau^{-}(x)+0} x$.

Let for $z \in M_{1}$,

$$
\tau(z)=\left\{\sup \left\{t>0: \text { for all } s, 0 \leqq s \leqq t, T^{s} z \in \partial M\right\}\right.
$$

if it is positive; otherwise $\left.\inf \left\{t>0: T^{t} z \in \partial M\right\}\right\}$.

Because of $\tau^{+}(x)<\infty \mu$-a.e. the transformation $T_{1} x=T^{\tau(x)+0} x, x \in M_{1}$, is defined that maps $M_{1}$ into itself. It is easy to see [CFS] that $T_{1}$ preserves the measure $\mu_{1}$, $d \mu_{1}=$ const $\cos \phi d r d \phi$, that is the projection of $\mu$ onto $M_{1}$, where const is the normalizing factor. So from the view of ergodic theory [CFS] we obtain the special representation of the flow $\left\{T^{t}\right\}$ by the automorphism $T_{1}$ of $M_{1}$ and the function $\tau(x), x \in M_{1}$. The differential of $T_{1}$ we denote by $D T_{1}$.

Let $t>0(t<0)$ be the moment of reflection from the boundary of the trajectory of a point $x$. We denote by $-T^{t} x$ the unit tangent vector such that $\pi\left(-T^{t} x\right)$ $=\pi\left(T^{t} x\right) \in \partial Q$ and $-T^{t} x$ has the direction opposite to the direction of $T^{t-0} x\left(T^{t+0} x\right)$. For $x \in M_{1}$ the unit tangent vector $-T_{1}^{n} x$ is defined in the same way.

It is known [CFS] that the measure (with respect to $\mu$ as well as with respect to $\mu_{1}$ ) of the set of all unit tangent vectors such that their trajectories intersect $V_{0}$ equals zero. We denote by $M^{\prime}$ a collection of all points $x \in M$ such that $T^{t} x$ is defined for any $t,-\infty<t<\infty$.

Let $M_{1}^{\prime}=M_{1} \cap M^{\prime}$, then $\mu_{1}\left(M_{1}^{\prime}\right)=1$. It is easy to see [CFS] that the ergodicity of the flow $\left\{T^{t}\right\}$ follows from the ergodicity of the dynamic system with discrete time generated by $T_{1}$.

Consider a curve $\tilde{\gamma} \subset Q$ that is of class $C^{2}$. By a framing $\gamma$ of the curve $\tilde{\gamma}$ one means a continuous section of the unit tangent bundle over $\tilde{\gamma}$ such that at each point $q \in \tilde{\gamma}$ a unit tangent vector $x \in \gamma, \pi(x)=q$, is orthogonal to $\tilde{\gamma}$ at the point $q$. According to this definition $\gamma$ corresponds to a beam of trajectories orthogonal to $\tilde{\gamma}$. It is clear that $\tilde{\gamma}$ has two framings. After choosing a framing we define a sign of the curvature of $\gamma$ at any of its points. If the curvature does not equal zero at all points of $\gamma$ then its value at a single point defines a framing uniquely. 
Let $\tilde{\gamma} \in Q$ be an arbitrary nonselfintersecting smooth curve and let $\gamma$ be its framing. Denote by $\kappa_{0}(x)$ a curvature of $\gamma$ at a point $x \in \gamma$. We assume that there are no points of $\gamma$ which do reflect from the boundary $\partial M$ during the time between 0 and $t$. It is not difficult to derive the relation (see [S1])

$$
\kappa_{t}(x)=\frac{\kappa_{0}(x)}{1+\kappa_{0}(x) t}
$$

where $\kappa_{t}(x)$ is the curvature of the curve $T^{t} \gamma$ at the point $T^{t} x$.

Hence if $\kappa_{0}(x)>0$ then $\kappa_{t}(x)>0$ for any $t>0$. In case $\kappa_{0}(x)<0$ one has $\kappa_{t}(x)<0$ for $0 \leqq t<1 /\left|\kappa_{0}(x)\right|$, the value $\kappa_{t}(x)$ equals infinity at the moment $t_{0}=1 /\left|\kappa_{0}(x)\right|$, and for $t>t_{0}$ the curvature $\kappa_{t}(x)$ is positive and decreases inversely proportional to $t-t_{0}$.

We shall call a smooth nonselfintersecting curve $\gamma$ convex (concave) at a point $x \in \gamma$ if $0<\kappa(x)<\infty(-\infty<\kappa(x)<0)$. A point $x$, where $\kappa(x)=0(\kappa(x)=\infty)$ will be called a flattening (conjugate) point. It is clear (see for instance [B1]) that convex, concave and flat curves generate divergent, convergent and parallel beams of trajectories (Fig. 1).

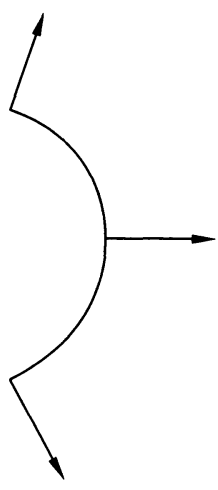

a)

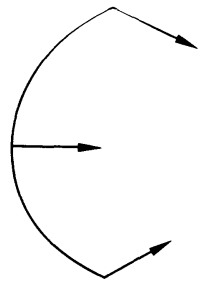

b)

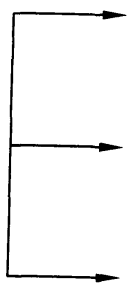

c)

The curvature of a curve suffers a jump at the moment of reflection. Denote by $\kappa_{-}(x)$ and $\kappa_{+}(x)$ the curvature of the curve $\gamma$ at the point $x \in \gamma, \pi(x) \in \partial Q$, at the moment just before and after the collision respectively. Then we obtain (see [BS]) that

$$
\kappa_{+}(x)=\kappa_{-}(x)+\frac{2 k^{(0)}(x)}{\cos \phi(x)}
$$


where $q=\pi(x), k^{(0)}(x)$ is the curvature of $\partial Q$ at the point $q, \phi(x)$ in the angle of incidence of the unit tangent vector $x\left(0 \leqq \phi(x) \leqq \frac{\pi}{2}\right)$.

Let now $x \in M_{1}$, and $T_{1}^{i} x \notin V_{0}$ for $0 \leqq i \leqq n$. We define

$$
\Lambda_{n}^{+}(x)=\prod_{i=1}^{n}\left|1+\kappa_{i-1}(x) \tau_{i}(x)\right|
$$

where $\tau_{i}(x)$ is the time between the $(i-1)^{\text {th }}$ and the $i^{\text {th }}$ reflections of the trajectory of $x$ from the boundary, $\tau_{1}(x)=\tau(x)$, and

$$
\kappa_{i}(x)=\frac{\kappa_{i-1}(x)}{1+\tau_{i}(x) \kappa_{i-1}(x)}+\frac{2 k_{i}^{(0)}(x)}{\cos \phi_{i}(x)},
$$

where $k_{i}^{(0)}(x)$ and $\phi_{i}(\mathrm{x})$ are the curvature of $\partial Q$ and the angle of incidence at the point of the $i^{\text {th }}$ reflection of the trajectory of $x$ from the boundary.

Analogously, we introduce a value

$$
\Lambda_{n}^{(-)}(x)=\prod_{i=1}^{n}\left|1+\bar{\kappa}_{i-1}(x) \bar{\tau}_{i-1}(x)\right|,
$$

where $\bar{\tau}_{i}(x)=\tau\left(-T_{1}^{-i} x\right)$ and

$$
\bar{\kappa}_{i}(x)=\frac{\bar{\kappa}_{i-1}(x)}{1+\bar{\tau}_{i-1}(x) \bar{\kappa}_{i-1}(x)}+\frac{2 \bar{k}_{i}^{(0)}(x)}{\cos \bar{\phi}_{i}(x)},
$$

where $k_{i}^{(0)}(x)$ is curvature of $\partial Q$ at the point $\pi\left(T_{1}^{-i} x\right)$ and $\bar{\phi}_{i}(x)$ is the corresponding angle of incidence.

The relations (3), (4), $\left(\left(3^{\prime}\right),\left(4^{\prime}\right)\right)$ do not determine completely the values of $\Lambda_{n}^{(+)}(x)$ $\left(\Lambda_{n}^{(-)}(x)\right)$. One has to fix also the curvature $\kappa_{0}(x)\left(\bar{\kappa}_{0}(x)\right)$ of a local beam of trajectories under consideration. According to formulas (1), (2), (4), (4') this curvature could be defined by fixing the value of the derivative $d \phi /\left.d r\right|_{x}$, $x=(r, \phi) \in M_{1}$.

For any integer $k$ we define $S_{k}=T_{1}^{k} S_{0}, V_{k}=T_{1}^{k} V_{0}$. The transformation $T_{1}\left(T_{1}^{-1}\right)$ has singularities on the set $S_{-1}=T_{1}^{-1} S_{0}\left(S_{1}=T_{1} S_{0}\right)$, where it is discontinuous and on the set $V_{-1}=T_{1}^{-1} V_{0}\left(V_{1}=T_{1} V_{0}\right)$, where it or its derivative is discontinuous.

Denote $\quad R_{-1}^{0}=R_{0} \cup S_{-1} \cup V_{-1}, \quad R_{1}^{0}=R_{0} \cup S_{1} \cup V_{1}, \quad R^{+}=\bigcup_{k=0}^{\infty} T_{1}^{k} R_{0}$, $R^{-}=\bigcup_{k=0}^{\infty} T_{1}^{-k} R_{0}$

It is easy to see that the set $S_{0}$ is given via the formula $\phi= \pm \frac{\pi}{2}$ on the cylinder (or on the collection of cylinders) $M_{1}$. The set $V_{0}$ forms a finite collection of vertical segments $r=\hat{r}_{i}, 1 \leqq i \leqq L$, where $\hat{r}_{i}$ is the coordinate of a singular point $\hat{q}_{i}$.

Let $\mathscr{M}^{+}\left(\mathscr{M}^{-}\right)$be a set consisting of all points $x \in M_{1}$ such that the positive (negative) semitrajectory of $T_{1}^{\hat{1}+(x)} x\left(T_{1}^{\hat{n}-(x)} x\right)$ has reflections from the boundary $\partial Q$ at $\partial Q^{0}$ for some integer $\hat{n}_{+}(x)<\infty\left(\hat{n}_{-}(x)<\infty\right)$. Denote $\mathscr{M}^{+} \cup \mathscr{M}^{-}=\mathscr{M}$. It is clear that expanding (contracting) cones $K^{(n)}(y)\left(K^{(s)}(y)\right)$ do not exist at the points $T_{1}^{\hat{n}_{+}(x)} x, \quad T_{1}^{\hat{n}_{+}(x)+1} x, \ldots\left(T_{1}^{\hat{n}_{-}(x)} x, \quad T_{1}^{\hat{n}_{-}(x)-1} x, \ldots\right)$. It is easy to see that $\mathscr{M} \backslash \overline{\mathscr{M}}^{+}\left(\mathscr{M} \backslash \overline{\mathscr{M}}^{-}\right)$is an open set.

We suppose that the following condition is fulfilled for billiards under consideration. 
G1. For point $x \in M_{1} \backslash \bar{M}$ such that $T_{1} x \notin R_{0}\left(T_{1}^{-1} x \notin R_{0}\right)$ there exist closed cones $K^{(u)}(x)\left(K^{(s)}(x)\right)$ in the tangent space $\mathscr{T}_{x} M_{1}$ to $M_{1}$ at the point $x$ such that $K^{(u)}(x)$ $\cap K^{(s)}(x)=0$,

$$
\begin{gathered}
D_{x} T_{1}\left(K^{(u)}(x)\right) \subseteq K^{(u)}\left(T_{1} x\right), \\
\left(D_{x} T_{1}^{-1}\left(K^{(s)}(x)\right) \subseteq K^{(s)}\left(T_{1}^{-1} x\right)\right),
\end{gathered}
$$

and there exist integers $k_{+}(x) \geqq 1, k_{-}(x) \geqq 1$ such that

$$
\begin{gathered}
D_{x} T_{1}^{k_{+}(x)}\left(K^{(u)}(x)\right) \subset K^{(u)}\left(T_{1}^{k_{+}(x)} x\right), \\
D_{x} T_{1}^{-k_{-}(x)}\left(K^{(s)}(x)\right) \subset K^{(s)}\left(T_{1}^{-k-(x)} x\right),
\end{gathered}
$$

where

$$
\begin{gathered}
K^{(u)}(x)=\left\{k_{1}^{(u)}(x) \leqq \frac{d \phi}{d r} \leqq k_{2}^{(u)}(x)\right\}, \\
K^{(s)}(x)=\left\{k_{1}^{(s)}(x) \leqq \frac{d \phi}{d r} \leqq k_{2}^{(s)}(x)\right\}, \\
0<k_{1}^{(u)}(x)<k_{2}^{(u)}(x)<\infty\left(-\infty<k_{1}^{(s)}(x)<k_{2}^{(s)}(x)<0\right) \\
\text { if } \pi(x) \in \partial Q^{+} \cup \partial Q^{0} \text { and }-\infty<k_{1}^{(u)}(x)<k_{2}^{(u)}(x)<0 \\
\left(0<k_{1}^{(s)}(x)<k_{2}^{(s)}(x)<\infty\right) \text { if } \pi(x) \in \partial Q^{-} .
\end{gathered}
$$

The cones $K^{(u)}(x)$ and $K^{(s)}(x)$ will be called expanding and contracting respectively. [One has to remark that $\left(5^{\prime}\right)$ means a strict inclusion.]

The next condition is the following one.

We shall call a $C^{1}$-smooth curve $\Sigma \subset M_{1}$ expanding (contracting) if for any point $y=(r, \phi) \in \Sigma$ one has $k_{1}^{(u)}(y) \leqq d \phi / d r \leqq k_{2}^{(u)}(y)\left(k_{1}^{(s)}(y) \leqq d \phi / d r \leqq k_{2}^{(s)}(y)\right)$. It follows from (5), ( $\left.5^{\prime}\right)$ that if $\Sigma$ is an expanding (contracting) curve and the transformation $T_{1}$ $\left(T_{1}^{-1}\right)$ is smooth on $\Sigma$ then $T_{1} \Sigma\left(T_{1}^{-1} \Sigma\right)$ is also expanding (contracting).

It is easy to see that to every expanding curve $\Sigma \subset \pi^{-1}\left(\partial Q^{+} \cup \partial Q^{0}\right)$ there corresponds a divergent beam of trajectories (Fig. 2a) and to every expanding curve $\Sigma \subset \pi^{-1}\left(\partial Q^{-}\right)$there corresponds a convergent beam of trajectories. A divergent beam would be divergent at all times up to the next reflection from the boundary. For a convergent beam of trajectories there exist different possibilities in this respect which are represented in Fig. $2 b$ and $c$.

We assume that billiards of our class satisfy the condition

G2. Each smooth component of the set $T^{n} R\left(T^{-n} R\right)$ is an expanding (contracting) curve, where $n>0$ is an integer.

The following property of billiards under consideration is their hyperbolicity.

G3. For almost every point $x \in M$ there exist local unstable manifold (LUM) $W^{(u)}(x)$ and local stable manifold (LSM) $W^{(s)}(x)$ such that both $W^{(u)}(x)$ and $W^{(s)}(x)$ are $C^{1}$ curves. Besides $T^{t}$ is continuous on $W^{(x)}(x)\left(W^{(s)}(x)\right)$ for all $t<0(t>0)$ and for any points $y_{1}, y_{2} \in W^{(u)}(x) \quad\left(y_{1}, y_{2} \in W^{(s)}(x)\right), \varrho\left(T^{t} y_{1}, \quad T^{t} y_{2}\right) \stackrel{t \rightarrow-\infty}{\longrightarrow} 0\left(\varrho\left(T^{t} y_{1}\right.\right.$, $\left.T^{t} y_{2}\right) \stackrel{t \rightarrow \infty}{\longrightarrow} 0$ ), where $\varrho(\cdot, \cdot)$ is a metric in $T^{t} W^{(u)}(x)\left(T^{t} W^{(s)}(x)\right)$ induced by the

Riemannian metric in $M$. 


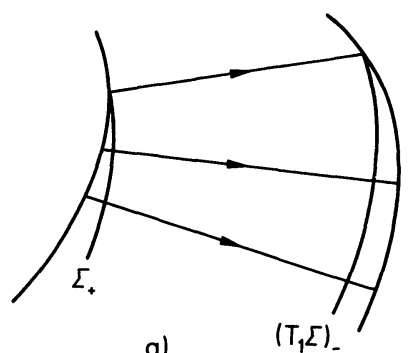

a)

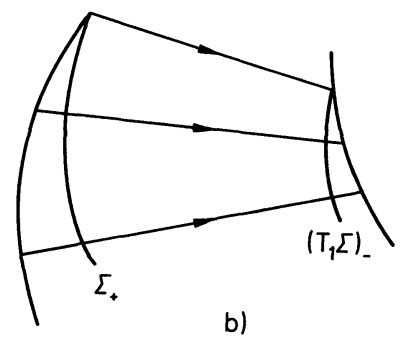

Fig. 2a-c

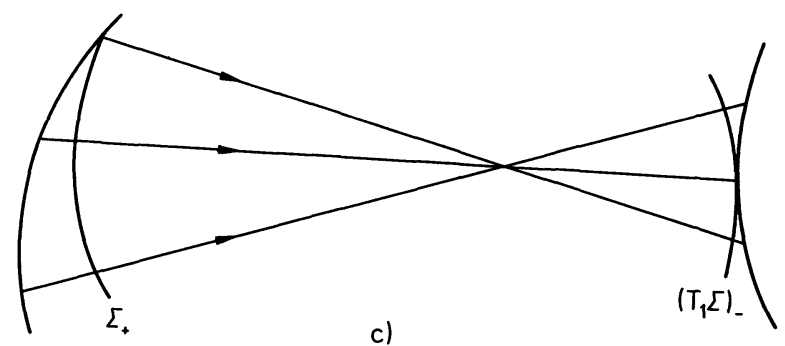

It is known [S1] that if G3 holds then there exist almost everywhere in $M_{1}$ LUM's $W_{1}^{(u)}(y)$ and LSM's $W_{1}^{(s)}(y)$ for the group of automorphisms $\left\{T_{1}^{n}\right\}$ that are projections on $M_{1}$ of LUM's and LSM's for $\left\{T^{t}\right\}$. It is easy to see that every LUM $W_{1}^{(u)}(y)$ is an expanding curve and every $\operatorname{LSM} W_{1}^{(s)}(y)$ is a contracting one.

The general theory of hyperbolic dynamical systems with singularities (see [KS]) states that for the billiards under consideration the foliations of $M_{1}$ into LUM and LSM are absolutely continuous.

We suppose that the following condition on defocussing holds.

G4. Let $\Sigma \subset \pi^{-1}\left(\partial Q^{-}\right)$be an expanding curve and let $T_{1} \mid \Sigma$ be continuous. Then for an arbitrary point $x \in \Sigma$ there exists a curve $\Sigma_{1} \subset \Sigma, x \in \Sigma_{1}$, such that the beam of trajectories generated by $\Sigma_{1}$ before the next reflection from the boundary is passing through a conjugate point, i.e. it becomes divergent.

The condition G4 means that for a beam of trajectories generated by an expanding curve $\Sigma \subset \pi^{-1}\left(\partial Q^{-}\right)$one has the situation that is represented in Fig. 2c.

Let us take an expanding curve $\Sigma \subset \pi^{-1}\left(\partial Q_{i}\right)$ that contains both its endpoints and consider a set $A(\Sigma)$ that consists of all closed segments $\left[x, T_{1} x\right]$. Consider now all curves $\gamma \subset M$ that are framings of curves $\tilde{\gamma}=\pi(\gamma) \subset Q$ orthogonal to all segments in $A(\Sigma)$. (We mean here a framing of $\tilde{\gamma}$ that is defined by the direction of trajectories corresponding to $\Sigma$.) The set of such curves will be denoted by $B(\Sigma)$. The curves $\gamma$ 
and $\tilde{\gamma}=\pi(\gamma)$ are closed sets. Suppose $\pi\left(T_{1} \Sigma\right) \subset \partial Q_{j}$. Let $\Sigma_{+}$be the curve $\gamma \subset B(\Sigma)$ that is the framing of the curve nearest ${ }^{1}$ to $\partial Q_{i}$ and let $\left(T_{1} \Sigma\right)_{-}$be the curve $\gamma_{1} \subset B(\Sigma)$ that is the framing of the curve $\tilde{\gamma}_{1}=\pi\left(\gamma_{1}\right)$ that is nearest to $\partial Q_{j}$. According to G4 this definition is a correct one.

Suppose $\gamma \subset M$ is the framing of the smooth nonselfintersecting curve $\tilde{\gamma}=\pi(\gamma)$ $\subset Q$ and denote by $\ell_{1}(\gamma)$ the length of $\tilde{\gamma}$ that is induced by the Euclidean metric in $Q$. We easily get from (1).

Proposition 1. Let $T^{t}$ be smooth in $\gamma$ and no point of $\gamma$ is reflected from $\partial Q$ during the time interval $(0, t)$. Then for any point $x \in \gamma$,

$$
\frac{d \ell_{1}\left(T^{t} x\right)}{d \ell_{1}(x)}=\left|1+\kappa_{0}(x) t\right|
$$

where $\kappa_{0}(x)$ is the curvature of $\gamma$ at the point $x$.

We put $\ell_{1}(\Sigma)=\ell_{1}\left(\Sigma_{+}\right)$for any expanding or contracting curve $\Sigma \subset M_{1}$.

We shall say that a contracting (expanding) cone $K^{(s)}(y)\left(K^{(u)}(y)\right)$ at a point $y$ is a perfect cone if

$$
\begin{aligned}
& \tilde{\Lambda}_{n}^{(-)}(y)=\min _{y \in \Sigma^{(s)}} \Lambda_{n}^{(-)}(y) \stackrel{n \rightarrow \infty}{\longrightarrow} \infty, \\
& \tilde{\Lambda}_{n}^{(+)}(y)=\min _{y \in \Sigma^{(u)}} \Lambda_{n}^{(+)}(y) \stackrel{n \rightarrow \infty}{\longrightarrow} \infty .
\end{aligned}
$$

The minimum in (6) is taken over all contracting (expanding) curves where the transformation $T_{1}^{-n}\left(T_{1}^{n}\right)$ is continuous. It is worthwhile to mention that by fixing $\Sigma^{(s)}\left(\Sigma^{(u)}\right)$ we determine simultaneously a value of $\bar{\kappa}_{0}(x)\left(\kappa_{0}(x)\right)$ in the formula for $\Lambda_{n}^{(-)}(x)\left(\Lambda_{n}^{(+)}(x)\right)$.

We introduce also the following property of strong defocussing.

G5. Suppose that at a point $x$ there exists a contracting cone and for some $k>0$ one has $T^{k} x \in \pi^{-1}\left(\partial Q_{i}^{-}\right), T^{k-1} x \notin \pi^{-1}\left(\partial Q_{i}^{-}\right), T^{k+i} x \in \pi^{-1}\left(\partial Q_{i}^{-}\right)$for $0 \leqq i<k_{0}, T^{k+k_{0}} x$ $\notin \pi^{-1}\left(\partial Q_{i}^{-}\right)$. Then $\frac{\tilde{\Lambda}_{k+k_{0}}^{(-)}(x)}{\tilde{\Lambda}_{k}^{(-)}(x)}>\tilde{\lambda}>1$. The analogous property holds for expanding cones if we consider the corresponding segment of the negative semitrajectory of $x$.

In view of G1-G5 and the invariance of $\mu_{1}$ the general theory of hyperbolic systems with singularities gives that the following property holds (see [KS, W]).

Proposition 2. Suppose there exist at a point $x$, contracting and expanding cones. Then one can find integers $n_{+}(x)>0\left(n_{-}(x)>0\right)$ such that for any expanding (contracting) curve $\Sigma^{(u)} \ni x\left(\Sigma^{(s)} \ni x\right)$ where the transformation $T_{1}^{n_{+}(x)}\left(T_{1}^{-n_{-}(x)}\right)$ is smooth we have (see (1)-(4)),

$$
\frac{\ell_{1}\left(T_{1}^{n_{+}(x)} \Sigma^{(u)}\right)}{\ell_{1}\left(\Sigma^{(u)}\right)}>1
$$

\footnotetext{
${ }^{1}$ The distance between two closed subsets $D_{1}$ and $D_{2}$ equals $\min _{q_{1} \in D_{1}, q_{2} \in D_{2}} d\left(q_{1}, q_{2}\right)$ where $d$ is the Euclidean metric in $Q$
} 
and correspondingly (see (1), (2), (3'), (4'))

$$
\frac{\ell_{1}\left(T_{1}^{-n_{-}(x)} \Sigma^{(s)}\right)}{\ell_{1}\left(\Sigma^{(s)}\right)}>1 .
$$

G1-G5 make the following proposition true as well.

Proposition 3. For every point $y \in M_{1}$ the cones $K^{(s)}(y)$ and $K^{(u)}(y)$ are perfect (if they exist).

In the following we usually speak about perfect cones.

Let $t_{0}=0<t_{1}<t_{2}<\ldots$ be the moments of consecutive reflections of the positive semitrajectory of a point $x \in M_{1}^{\prime}$ from the boundary.

Denote

$$
\begin{aligned}
& \kappa^{(s)}(x)=-\frac{1}{-}, \\
& \tau_{1}(x)+\frac{1}{-} \\
& \frac{2 k_{1}^{(0)}(x)}{\cos \phi_{1}(x)}+-1 \\
& \tau_{2}(x)+\frac{1}{-} \\
& \frac{2 k_{2}^{(0)}(x)}{\cos \phi_{2}(x)}+\frac{1}{\tau_{3}(x)}+\ldots
\end{aligned}
$$

where $\tau_{i}(x)=t_{i}-t_{i-1}, k_{i}^{(0)}(x)$ and $\phi_{i}(x)$ are the curvature of $\partial Q$ and the angle of incidence at the point of the $i^{\text {th }}$ reflection of the trajectory of $x$ from the boundary.

It is known [S1] that the LSM at the point $x$ is given by the following equation:

$$
\frac{d \phi}{d r}=\kappa^{(s)}(x) \cos \phi(x)-k^{(0)}(x)
$$

Consider $x^{\prime}=x-2(x, n(q)) n(q)$, where $q=\pi(x)$. Then the LUM at the point $x$ has the form [S1]

$$
\frac{d \phi}{d r}=\kappa^{(u)}(x) \cos \phi(x)+k^{(0)}(x)
$$

where $\kappa^{(u)}(x)=-\kappa^{(s)}\left(x^{\prime}\right)$.

The relations (8) and (9) can be derived [S1] from formulas (1), (2), and (4).

We shall assume that the following condition is fulfilled for the billiards under consideration.

G6. If the positive (negative) trajectory of a point $x \in M_{1}$ does not intersect with $R$ then $\kappa^{(s)}(x)\left(\kappa^{(u)}(x)\right)$ is continuous at $x$. Moreover for every $\varepsilon>0$ there exist a positive integer $n_{0}=n_{0}(\varepsilon)$ and a suitably small neighborhood $U_{\varepsilon}=U_{\varepsilon}(x)$ such that for $n>n_{0}(\varepsilon)$ and for every $y \in U_{\varepsilon} \cap M^{\prime}$ and every expanding curve $\gamma \ni\left(T^{n} y\right)^{\prime}$,

$$
\left|\kappa^{(s)}(x)-\kappa_{T_{\gamma}^{(s)}}^{(y)}\right|<\varepsilon,
$$


where

$$
\begin{aligned}
& \kappa_{T^{n} \gamma}^{(s)}(y)=-\frac{1}{-} . \\
& \tau_{1}(y)+\frac{1}{-} \\
& \frac{2 k_{1}^{(0)}(y)}{\cos \phi_{1}(y)}+\frac{1}{\tau_{2}(y)+\longrightarrow} \\
& \frac{2 k_{2}^{(0)}(y)}{\cos \phi_{2}(y)}+\ldots+\frac{1}{\tau_{n}(y)+\frac{1}{\kappa_{\gamma}\left(\left(T_{y}^{n}\right)^{\prime}\right)}}
\end{aligned}
$$

Here $\kappa_{\gamma}\left(\left(T^{n} y\right)^{\prime}\right)$ is the curvature of $\gamma$ at the point $\left(T^{n} y\right)^{\prime}$.

Denote $R_{-1}=S_{-1} \cup V_{-1}, R_{1}=S_{1} \cup V_{1}$. We shall formulate now the last condition (see [SC, KSS2]).

A. There exists a perfect cone $K^{(s)}(x)\left(K^{(u)}(x)\right)$ for almost every point $x \in R_{-1}^{0}$ $\left(x \in R_{1}^{0}\right)$ (with respect to a measure induced by the Euclidean metric on $R_{-1}^{0}$ and $\left.R_{1}^{0}\right)$.

Theorem 1. Let the billiard satisfy conditions G1-G6 and A. Consider a point $x_{1} \in M_{1}^{\prime}$ such that its trajectory $\left\{T_{1}^{n} x_{1}\right\},-\infty<n<\infty$, is not tangent to $\partial Q$ for all $n$ and the cones $K^{(u)}\left(x_{1}\right)$ and $K^{(s)}\left(x_{1}\right)$ are perfect ones. Then there exists a neighborhood $U_{1}\left(x_{1}\right)$ belonging mod 0 to one ergodic component of the dynamical system under consideration.

\section{Proof of Theorem 1}

For a point $x \in M$ by the length $L(x)$ of the segment $\left(\pi(x), \pi\left(T^{\tau(x)}(x)\right)\right.$ we shall understand the length of free path of $x$. If $Q$ is a bounded domain in $\mathbb{R}^{2}$ then $L(x)$ $<L_{0}(Q)<\infty$ for all points $x \in M$. The set $R$ where $T_{1}$ has discontinuities consists in this case of a finite number of smooth components.

If $Q \subset \mathbb{T}^{2}$ then in order to get the same situation we shall use the following procedure (see [SC]). Let $\mathrm{T}^{2}$ be obtained by gluing together the opposite sides of the rectangle $K$. Consider the billiard problem in the domain with boundary $\partial Q$ $\cup \partial K$. Inside this domain the motion is free with unit velocity. The reflections from $\partial Q$ are usual elastic reflections but when the moving point reaches $q \in \partial K$ then it instantly jumps to the point $q^{\prime} \in \partial K$ that corresponds to $q$ by the gluing and the further motion is going in the same direction (i.e. parallel to the previous segment of this trajectory that ended at $q$ ). We shall denote the corresponding derived automorphism of this extended boundary $\partial Q \cup \partial K$ into itself also by $T_{1}$. We preserve also the notation of phase space as $M_{1}$ and $\partial Q$ for $\pi\left(M_{1}\right)$. The invariant measure $\mu_{1}$ of the automorphism $T_{1}$ has the density $d \mu_{1}(x)=$ const $|(n(q), x)| d r d \phi$, where $q=\pi(x) \in \partial Q \cup \partial K$.

Let us now take a straight segment $\left[\pi x, \pi\left(T_{1}^{-i} x\right)\right]$ that has its endpoints in two regular components of the extended boundary $\partial Q$ and include it in a continuous family of parallel segments that have their endpoints in the same regular components of $\partial Q$ (Fig. 3). We denote by $\frac{1}{2} z(x)$ the distance from $\left[\pi x, \pi\left(T_{1}^{-1} x\right)\right]$ to 
Fig. 3

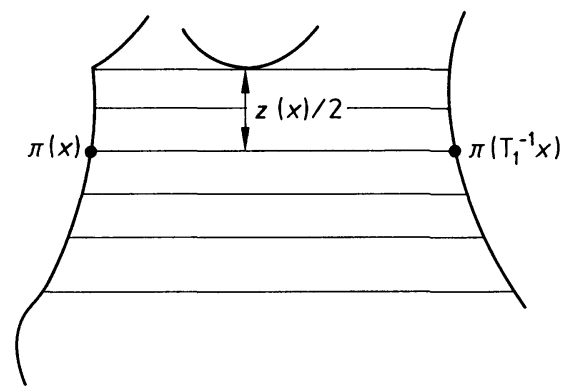

the nearest boundary segment of this family. It is clear that either such a boundary segment is tangent to $\partial Q$ or it contains a singular point of $\partial Q$ (Fig. 3).

In complete analogy to [SC] and [KSS2] one can prove

Lemma 1. For any arbitrary $\varepsilon>0$,

$$
\mu_{1}\left\{x \in M_{1}: z(x)<\varepsilon\right\}<c_{1} \varepsilon,
$$

where $c_{1}=c_{1}(Q)$.

An open quadrilateral $G$ will be called a parallelogram if one pair of its opposite sides consists of expanding curves and the other consists of contracting curves. The expanding (contracting) part of the boundary of $G$ will be denoted by $\Gamma^{(u)}(G)$ $\left(\Gamma^{(s)}(G)\right), \Gamma(G)=\Gamma^{(u)}(G) \cup \Gamma^{(s)}(G)$. A parallelogram $G$ is called $u$-inserted (s-inserted) in a parallelogram $G_{1}$ if $G \subset G_{1}$ and $\Gamma^{(s)}(G) \subset \Gamma^{(s)}\left(G_{1}\right)\left(\Gamma^{(u)}(G) \subset \Gamma^{(u)}\left(G_{1}\right)\right)$. It is clear that any parallelogram $G$ has a (nonunique) partition into expanding (contracting) curves with endpoints in $\Gamma^{(s)}(G)\left(\Gamma^{(u)}(G)\right)$.

Let $U\left(x_{1}\right)$ be an open neighborhood of $x_{1}$. Consider a family of finite coverings $B^{(\delta)}=\left\{G_{1}^{(\delta)}, \ldots, G_{m}^{(\delta)}\right\}, m=m(\delta)$, of $U_{1}\left(x_{1}\right)$, where every $G_{1}^{(\delta)}, 1 \leqq i \leqq m$, is a parallelogram and the parameter $\delta$ will tend to zero in what follows.

The sets $G_{i}^{\delta}$ can be considered as images of the standard unit square under inhomogeneous linear mappings $\mathbb{R}^{2} \rightarrow U\left(x_{1}\right)$ where linearity is defined in terms of some fixed coordinate system in $U\left(x_{1}\right)$.

A family $B^{(\delta)}$ will be called a regular one if the following conditions are satisfied:

1. the centers $y_{i}^{\delta}$ of $G_{i}^{(\delta)}$ (with respect to the fixed coordinate system) have LSM and LUM and, moreover, the tangents to $W^{(u)}\left(y_{i}^{\delta}\right)$ and $W^{(s)}\left(y_{i}^{\delta}\right)$ are parallel (according to coordinate system) to the corresponding sides of $G_{i}$.

2. if $G_{i}^{(\delta)} \cap G_{j}^{(\delta)} \neq \emptyset$ then $\mu_{1}\left(G_{i}^{(\delta)} \cap G_{j}^{(\delta)}\right) \geqq C_{1} \delta^{2}$, where $C_{1}>0$ does not depend on $\delta$. 3. any point $y \in U_{1}\left(x_{1}\right)$ does belong to not more than four different parallelograms $G_{i}^{(\delta)}$ for any fixed $\delta$.

4. the centers $y_{i}^{\delta}$ of parallelograms $G_{i}^{(\delta)}, i=1, \ldots, m(\delta)$, constitute asymptotically as $\delta \rightarrow 0$ a linear lattice (with respect to the fixed coordinate system in $U\left(x_{1}\right)$ ).

It is clear that regular coverings exist. We shall consider in what follows such regular coverings that contain long strips of parallelograms that extend into directions corresponding to expanding and contracting curves.

The crucial point in the proof of Theorem 1 is the following 
Lemma 2. For any $\delta<\delta_{0}\left(x_{1}\right)$ the parallelograms of the regular covering $\left\{G_{i}^{(\delta)}\right\}$ could be divided into two groups $U_{0}^{(\delta)}$ and $U_{1}^{(\delta)}$ such that

(a) let $U^{(u)}$ be a neighborhood of radius $\varepsilon_{1} \delta$ of the two expanding sides of $a$ parallelogram $G_{i}^{(\delta)} \subset U_{1}^{(\delta)}$. The constant $\varepsilon_{1}$ does not depend on $\delta$ and could be made arbitrarily small by taking smaller neighborhoods $U_{1}\left(x_{1}\right)$. Consider now all points $z \in U^{(u)}$ that $W_{1}^{(u)}(z)$ intersect both contracting sides of a parallelogram $G_{i}^{(\delta)}$. Then the set that consists of all these points has positive measure.

(b) the measure of the union of all parallelograms belonging to $U_{0}^{(\delta)}$ equal to $\delta \phi_{1}(\delta)$, where $\phi_{1}(\delta) \rightarrow 0$ as $\delta \rightarrow 0$.

The analogous assertion holds if in condition (a) we consider a contracting side of $G_{i}^{(\delta)}$ instead of an expanding one.

We will show how Theorem 1 follows from Lemma 2. It is quite analogous to [S2, S3, SC, KSS2].

As was mentioned above the proof of Theorem 1 is based on Hopf's idea [H]. Let us mention first that every LSM (LUM) belongs mod0 to one ergodic component. It was proved in [S2] that the following assertion follows from Lemma 2.

Lemma 3. There exists a collection of parallelograms $U_{2}^{(\delta)} \subset U_{1}^{(\delta)}$ such that

(a2) $\mu_{1}\left(U_{1}\left(x_{1}\right) \underset{G_{i}^{(\delta)} \subset U_{2}^{(\delta)}}{\bigcup} G_{i}^{(\delta)}\right)<\phi_{2}(\delta)$, where $\phi_{2}(\delta) \rightarrow 0$ as $\delta \rightarrow 0$.

Furthermore according to $\mathrm{G} 3$, for almost every pair of points $x^{\prime}, x^{\prime \prime} \in U_{1}\left(x_{1}\right)$ there exist LSM $W_{1}^{(s)}\left(x^{\prime}\right)$ and LUM $W_{1}^{(u)}\left(x^{\prime \prime}\right)$ and $\ell_{1}\left(W_{1}^{(s)}\left(x^{\prime}\right)\right)-\delta^{\prime}>0, \ell_{1}\left(W_{1}^{(u)}\left(x^{\prime \prime}\right)\right)$ $-\delta^{\prime \prime}>0$. Therefore one can find $\delta<\min \left\{\delta^{\prime}, \delta^{\prime \prime}\right\}$ such that $W_{1}^{(s)}\left(x^{\prime}\right)$ and $W_{1}^{(u)}\left(x^{\prime \prime}\right)$ to intersect the parallelograms belonging to $U_{2}^{(\delta)}$. Hence, in view of a) in the formulation of Lemma 2, one can find a so-called Hopf chain, i.e. a collection $W_{1,1}^{(s)}$, $W_{1,2}^{(u)}, W_{1,3}^{(s)}, \ldots, W_{1, k}^{(u)}$ of LSM and LUM such that $x^{\prime} \in W_{1,1}^{(s)}, x^{\prime \prime} \in W_{1, k}^{(u)}$ and $W_{1, i}^{(s)}$ $\cap W_{i, i+1}^{(u)} \neq \emptyset$ or $W_{1, i}^{(u)} \cap W_{1, i+1}^{(s)} \neq \emptyset$ for all $i=1,2, \ldots, k-1$. Thus the points $x^{\prime}$ and $x^{\prime \prime}$ belong to the same ergodic component.

Proof of Lemma 2. Recall that the transformation $T_{1}$ is pointwise smooth. Making use of the procedure of the extension of the boundary we obtained, in the case when $Q \subset \mathbb{T}^{2}$, that $T_{1}^{k}$ has singularities in the set $\bigcup_{j=0}^{-k+1} T_{1}^{j} R_{0}$ which is the union of a finite number of curves for any integer $k$. In what follows we shall refer to these curves as to (generalized) discontinuity curves of $T_{1}^{k}$.

Denote by $U_{00}^{(\delta)}$ the union of all parallelograms $G_{i}^{(\delta)}$ which intersect at least two discontinuity curves of the transformation $T_{1}^{F(\delta)}$. The next lemma follows from Lemma 4.6 in [KSS2] (see also [S3]).

Lemma 4. There exist functions $F(\delta), F(\delta) \stackrel{\delta \rightarrow 0}{\longrightarrow} \infty$ and $\phi_{3}(\delta), \phi_{3}(\delta) \stackrel{\delta \rightarrow 0}{\longrightarrow} 0$ such that $\mu_{1}\left(U_{00}^{(\delta)}\right)<\delta \phi_{3}(\delta)$.

Proof. We shall include $U_{00}^{(\delta)}$ into the set $U_{0}^{(\delta)}$. 
Let $\Sigma^{(u)}(x) \subset M_{1}$ be an expanding curve. Denote by $x_{+}\left(x_{-}\right)$the point on the trajectory of $x$ closest to $x$ that belongs to $\Sigma_{+}^{(u)}(x)\left(\Sigma_{-}^{(u)}(x)\right)$. An analogous object could be considered for a contracting curve $\Sigma^{(s)}(x) \subset M_{1}$.

We take a point $y \in M_{1}^{\prime} \backslash \overline{\mathscr{M}}^{-}$, an integer $n>0$ and $\delta>0$. Consider all expanding curves $\Sigma^{(u)}\left(T_{1}^{-n} y\right)$ that contain the point $T_{1}^{-n} y=y_{1}$ such that $T_{1}^{n} \mid \Sigma^{(u)}\left(T_{1}^{-n} y\right)$ is smooth and that both distances from the point $q_{+}\left(T_{1}^{-n} y\right)=\pi\left(\left(T_{1}^{-n} y\right)_{+}\right)$to endpoints of the curve $\pi\left(\Sigma_{+}^{(u)}\left(T_{1}^{-n} y\right)\right)$ do not exceed $\delta$.

Denote by $\lambda_{n, \delta}^{(-)}(y)$ the minimal coefficient of expansion at any point of such curves that arise by the transformation of $\Sigma_{+}^{(u)}\left(T_{1}^{-n} y\right)$ into $\left(T_{1}^{n} \Sigma^{(u)}\left(T_{1}^{-n} y\right)\right)_{-}$. By $\lambda_{n, \Sigma}^{(-)}$ we denote the minimal coefficient of expansion at points of a fixed curve $\Sigma$.

Lemma 5. If the negative semitrajectory of the point $y \in M_{1}^{\prime} \backslash \mathscr{M}^{-}$does not touch $\partial Q$ then $\lambda_{n, \delta}^{(-)}(y)$ increases monotonically as $\delta \rightarrow 0$ and has the limit

$$
\Lambda_{n}^{(-)}(y)=\prod_{i=1}^{n}\left|1+\bar{\kappa}_{i-1}^{\prime}\left(y_{1}\right) \bar{\tau}_{i}\left(y_{1}\right)\right|,
$$

where the values $\Lambda_{n}^{(-)}(y)$ were defined in formula $\left(3^{\prime}\right), \bar{\kappa}_{0}^{\prime}\left(y_{1}\right)=k_{1}^{(0)}\left(y_{1}\right)+\frac{k^{(0)}\left(y_{1}\right)}{\cos \phi\left(y_{1}\right)}$ if $\pi\left(y_{1}\right) \in \partial Q^{0} \cup \partial Q^{+}, \bar{\kappa}_{0}^{\prime}\left(y_{1}\right)=k_{2}^{(0)}\left(y_{1}\right)+k^{(0)}\left(y_{1}\right) / \cos \phi\left(y_{1}\right)$, if $\pi\left(y_{1}\right) \in \partial Q^{-}$and the values $\bar{\kappa}_{i}^{\prime}(y)$ were defined for $i>0$ by the recurrence relation $\left(4^{\prime}\right)$.

Proof. In view of the definition of expanding curves the beam of trajectories that correspond to $\Sigma^{(u)}\left(T_{1}^{-n} y\right)$ is divergent if $\pi\left(T_{1}^{-n} y\right) \in \partial Q^{+} \cup \partial Q^{0}$ and is convergent if $\pi\left(T_{1}^{-n} y\right) \in \partial Q^{-}$. In the first case it follows from (1)-(4) that the minimal coefficient of expansion along the trajectory of a point $z \in \Sigma^{(u)}\left(T_{1}^{-n} y\right)$ corresponds to an expanding curve $\Sigma^{(u)}\left(T_{1}^{-n} y\right)$ that has minimal curvature at $z$. Furthermore if $\pi\left(T_{1}^{-n} y\right) \in \partial Q^{-}$then, as we explain below, one can obtain from (1)-(4) and G4 that the minimal coefficient of expansion is in the curve $\Sigma^{(u)}\left(T_{1}^{-n} y\right)$ that has minimal modulus of curvature. The only case which we have to consider corresponds to a reflection from $\partial Q^{-}$such that the next reflection is from $\partial Q^{+}$.

Consider two local convergent beams of trajectories that have reflections from $M_{1}^{-}$at the same point $x$. Let the curvatures of these beams after these reflections be $\kappa_{1}^{\prime}(x)$ and $\kappa_{1}^{\prime \prime}(x)$ with $\left|\kappa_{1}^{\prime}(x)\right|<\left|\kappa_{1}^{\prime \prime}(x)\right|$. (Recall that both values $\kappa_{1}^{\prime}(x)$ and $\kappa_{1}^{\prime \prime}(x)$ are negative.) Then the coefficient of expansion under the action of $T$ equals $\lambda_{1}^{\prime}=\tau_{2}^{\prime} / \tau_{1}^{\prime}$ for the first beam and equals $\lambda_{1}^{\prime \prime}=\tau_{2}^{\prime \prime} / \tau_{1}^{\prime \prime}$ for the second one, where $\tau_{1}^{\prime}=\left|\kappa_{1}^{\prime}(x)\right|^{-1}$, $\tau_{1}^{\prime \prime}=\left|\kappa_{1}^{\prime \prime}(x)\right|^{-1}, \tau_{1}^{\prime}+\tau_{2}^{\prime}=\tau_{1}^{\prime \prime}+\tau_{2}^{\prime \prime}=\tau(x)$. So $\tau_{1}^{\prime \prime}<\tau_{1}^{\prime}, \tau_{2}^{\prime \prime}>\tau_{2}^{\prime}$. We have $\kappa_{-}^{\prime}(T x)=\left(\tau_{2}^{\prime}\right)^{-1}$, $\kappa_{-}^{\prime \prime}(T x)=\left(\tau_{2}^{\prime \prime}\right)^{-1}$. So according to (1), (2), we get

$$
\begin{aligned}
& \lambda_{2}^{\prime}=\left(\kappa_{-}^{\prime}(T x)+2 \frac{k^{(0)}(T x)}{\cos \phi(T x)}\right) \tau(T x)+1, \\
& \lambda_{2}^{\prime \prime}=\left(\kappa^{\prime \prime}(T x)+2 \frac{k^{(0)}(T x)}{\cos \phi(T x)}\right) \tau(T x)+1 .
\end{aligned}
$$

Therefore $\lambda_{1}^{\prime} \lambda_{2}^{\prime}<\lambda_{1}^{\prime \prime} \lambda_{2}^{\prime \prime}$. If $T_{1}^{2} x, \ldots, T^{n} x \in \partial Q^{-}$then it is easy to verify by induction that the needed assertion is true, i.e. the minimal expansion coefficient corresponds to the curve that has minimal (in modulus) initial curvature.

It follows from the definition of the curves $\Sigma_{+}^{(u)}$ that $\lambda_{n, \delta}^{(-)}(y)$ increases monotonically as $\delta \rightarrow 0$. Hence there exists a limit $\lambda_{n, 0}^{(-)}(y)=\lim _{\delta \rightarrow 0} \lambda_{n, \delta}^{(-)}(y)$ that is 
defined by the relation given in the formulation of lemma. Thus Lemma 5 is proven.

It is easy to see that the analogous assertion holds if the positive semitrajectory of the point $y \in M_{1}^{\prime} \backslash \mathscr{M}^{+}$does not touch $\partial Q$. In this case one has to introduce the corresponding values $\lambda_{n, \delta}^{(+)}(y)$ and to use in the formulation of Lemma 5 , the relation (4) instead of $\left(4^{\prime}\right)$.

According to the conditions of Theorem 1 one can find $n_{0}=n_{0}\left(x_{1}\right)$ such that the following property holds:

$(\alpha)$ Let $U_{1}\left(x_{1}\right)$ be a neighborhood of $x_{1}$ where $T_{1}^{n_{0}}$ is smooth. Take now a point $x \in U_{1}\left(x_{1}\right)$. Then for any expanding curve $\Sigma^{(u)}$ containing $\left(-T_{n}^{n_{0}} x\right)$ the curve $\Sigma_{+}^{(u)}$ under the transformation into $\left(T_{1}^{n_{0}} \Sigma^{(u)}\right)_{-}$expands by a factor not less than $\Lambda>1$, where $\Lambda=\Lambda\left(x_{1}\right)$ does not depend on $x$ and $\Sigma^{(u)}$.

In order to show it we mention first that the cone $K^{(u)}\left(x_{1}\right)$ is a perfect one. Therefore $x_{1} \notin \mathscr{M}^{+}$. Further because dispersing components of the boundary $\partial Q$ intersect transversally there exists an integer $\hat{n}_{1}>0$ such that for any expanding curve $\Sigma^{(u)}$ the minimal coefficient of expansion $\lambda_{n, \Sigma^{(u)}}^{(-)}>\lambda^{\prime}>1$ if $T^{k} \Sigma^{(u)} C M_{1}^{+}$for $k=0,1, \ldots, n, n>\hat{n}_{1}$ (see [BS]). Then according to $\mathrm{G} 4$ and the condition of strong defocussing G5, for any expanding curve $\Sigma^{(u)} \subset M_{1}^{\prime}$ there exists an integer $\hat{n}_{2}>0$ such that $\lambda_{n, \Sigma^{(u)}}^{(-)}>\lambda^{\prime \prime}>1$ if $n>\hat{n}_{2}$. Hence a neighborhood $U_{1}\left(x_{1}\right)$ with the needed properties exists.

We choose now a neighborhood $U_{1}\left(x_{1}\right)$ small enough that $T_{1}^{n} y \notin U_{1}\left(x_{1}\right)$ for all $y \in U_{1}\left(x_{1}\right), 0 \leqq n<n_{0}$. [If the point $x_{1}$ is periodic then we can make the same choice since $n_{0}\left(x_{1}\right)$ does not exceed the period $x_{1}$.]

Consider now the set

$$
U_{1}^{(0)}=\left\{y \in U_{1}\left(x_{1}\right): z\left(T_{1}^{n} y\right) \geqq \delta \lambda_{n, \delta}^{(+)-1}(y) \text { for all } n>0\right\} .
$$

Lemma 6. There exists a number $C_{2}>0$ such that for any point $y \in U_{1}^{(0)}$ the length of the LSM $W_{1}^{(s)}(y)$ is bounded from below by $C_{2} \delta$.

Proof. Denote by $r(y), y \in M$, the length in the metric $\ell_{1}$ of the arc from the point $\pi\left(y_{+}\right)$to the nearest endpoint of $\pi\left(\left(W_{1}^{(s)}(y)\right)_{+}\right)$. We put $r(y)=0$ if $y$ does not have an LSM.

The proof of the following statement is completely analogous to the proof of Lemma 4 in [SC] (see also Lemma 5.4 in [KSS2]).

Lemma 7. If $y \in U_{1}^{(0)}$ then $r(y) \geqq \delta$.

Proof. Take any expanding curve $\Sigma^{(n)} \ni-T_{1}^{n} y$ such that $\Sigma_{+}^{(u)}$ has its center at the point $\left(-T_{1}^{n} y\right)_{+}$and its $\ell_{1}$-length equals $\min \left\{\delta, z\left(T_{1}^{n} y\right)\right\}$. The mapping $\hat{T}_{n}$ that transforms $\Sigma_{+}^{(u)}$ into $\left(T_{1}^{n} \Sigma^{(u)}\right)_{-}$is pointwise smooth. Let $\Sigma_{+, 0}^{(n)} \ni T_{1}^{-n} y$ be a smooth component of a curve $\Sigma_{+}^{(n)}$ where this mapping acts smoothly and $\tilde{\Sigma}_{+, 0}^{(n)}=\pi\left(\Sigma_{+, 0}^{(n)}\right)$. In view of the definition of $\lambda_{n, \delta}^{(+)}(y)$, the $\ell_{1}$-length of the curve $\widetilde{\Sigma}_{+, 0}^{(n)}$ increases by a factor not less than $\lambda_{n, \delta}^{(+)}(y)$ under the action of $\hat{T}_{n}$.

Let $r_{n}(y)$ be the $\ell_{1}$-distance from $\pi\left((-y)_{-}\right)$to the nearest endpoint $\pi\left(\left(u^{(n)}\right)_{-}\right)$of the curve $\pi\left(\widehat{T}_{n} \Sigma_{+, 0}^{(n)}\right)$. Suppose $r_{n}(y)<\delta$ for some $n \geqq 0$. If $u^{(n)}=\widehat{T}_{n} u_{0}^{(n)}$, where $\pi\left(u_{0}^{(n)}\right) \in \partial \widetilde{\Sigma}_{+, 0}^{(n)}$ then $z\left(\left(-T_{1}^{n} y\right) \geqq \delta \lambda_{n, \delta}^{(+)}{ }^{-1}\right.$ and $r_{n}(y) \geqq z\left(-T_{1}^{n} y\right) \lambda_{n, \delta}^{(+)} \geqq \delta$.

In the opposite case one can find an integer $k, 0<k<n$, such that $T_{1}^{k} u_{0}^{(n)} \in S_{0}$ $\cup V_{0}$. So $z\left(T_{1}^{k} y\right) \geqq \delta \lambda_{k, \delta}^{(+)}(y)$ as $y \in U_{1}^{(0)}$. Hence $r_{n}(y) \geqq z\left(T_{1}^{k} y\right) \lambda_{k, \delta}^{(+)}(y) \geqq \delta$. Therefore 
$r_{n}(y) \geqq \delta$ in any case. In view of the relation $W_{1}^{(s)}(y)=\lim _{n \rightarrow \infty}-\left(\pi_{1}^{+}\left(\widehat{T}_{n} \Sigma_{+, 0}^{(n)}\right)\right)$ Lemma 7 is proven.

The transformation $\pi_{1}^{+}$is smooth at the point $\left(x_{1}\right)_{-}$. Therefore we can choose $C_{2}$ such that $W_{1}^{(s)}(y)$ has length not less than $C_{2} \delta$. Lemma 6 is proven.

Introduce the set $U_{0}=U_{1}\left(x_{1}\right) \backslash U_{1}^{(0)}$. Then $U_{0}=U_{0,1} \cup U_{0,2} \cup \ldots$, where $U_{0, n}$ $=\left\{y \in U_{1}\left(x_{1}\right): z\left(T_{1}^{n} y\right) \leqq \delta\left(\lambda_{n, \delta}^{(+)}(y)\right)^{-1}\right\}$. (We shall omit the index $(+)$ in $\lambda_{n, \delta}^{(+)}$in what follows.) We have $U_{0, n} \subseteq U_{0, n, 0} \cup U_{0, n, 1} \cup \ldots$, where

$$
U_{0, n, m}=\left\{y \in U_{0, n}: \log _{\Lambda} \lambda_{n, \delta}^{-1}(y) \in[m, m+1)\right\} .
$$

Hence

$$
\mu_{1}\left(U_{0}\right) \leqq \sum_{n=1}^{\infty} \sum_{m=0}^{\infty} \mu_{1}\left(U_{0, n, m}\right)=\sum_{m=0}^{\infty} \sum_{n=1}^{\infty} \mu_{1}\left(T_{1}^{n} U_{0, n, m}\right) .
$$

In the same way as Lemma 5 in [SC] and Lemma 6.3 in [KSS2], one can prove

Lemma 8. For any $m \geqq 0,0 \leqq n_{1}<n_{2}$, the sets $T_{1}^{n_{1}} U_{0, n_{1}, m}$ and $T_{1}^{n_{1}} U_{0, n_{2}, m}$ do not intersect.

In fact the neighborhood $U_{1}\left(x_{1}\right)$ satisfies the condition $(\alpha)$. Therefore if there exists $z \in T_{1}^{n_{1}} U_{0, n_{1}, m} \cap T_{1}^{n_{2}} U_{0, n_{2}, m}$, then $T_{1}^{-n_{1}} z \in U_{0, n_{1}, m} \subset U_{1}\left(x_{1}\right), T^{-n_{2}} z \in U_{0, n_{2}, m}$ $C U_{1}\left(x_{1}\right)$ and also $\lambda_{n_{1}, \delta}\left(T^{-n_{1}} z\right) \in\left[\Lambda^{m}, \Lambda^{m+1}\right], \lambda_{n_{2}, \delta}\left(T^{-n_{2}} z\right) \in\left[\Lambda^{m}, \Lambda^{m+1}\right]$. But this contradicts $(\alpha)$ because the minimal expansion coefficient of the transformation $T^{n_{2}-n_{1}}$ on any expanding curve $\Sigma \subset U_{1}\left(x_{1}\right)$ is larger than $\Lambda$.

According to Lemmas 8 and 1 we can write

$$
\sum_{n=1}^{\infty} \mu_{1}\left(T_{1}^{n} U_{0, n, m}\right)=\mu_{1}\left(\bigcup_{n=1}^{\infty} T_{1}^{n} U_{0, n, m}\right) \leqq \mu_{1}\left\{y \in \partial M: z(y) \leqq \delta \lambda^{m}\right\} \leqq c_{2}\left(x_{1}\right) \delta \lambda^{m}
$$

(here $\lambda=\Lambda^{-1}$ ).

Equations (9) and (10) imply that

$$
\mu_{1}\left(U_{0}\right) \leqq \sum_{m=0}^{\infty} c_{2}\left(x_{1}\right) \delta \lambda^{m}=c_{3}\left(x_{1}\right) \delta .
$$

Consider $U_{0, \alpha}=\bigcup_{n=0}^{F(\delta)} U_{0, n}, U_{0, \omega}=\bigcup_{n=F(\delta)+1}^{\infty} U_{0, n}$. We shall show that

$$
\mu_{1}\left(U_{0, \omega}\right) \leqq \delta \phi_{4}(\delta),
$$

where $\phi_{4}(\delta) \rightarrow 0$ as $\delta \rightarrow 0$.

The following statement is proved in [SC] as Lemma 6 and in [KSS2] as Lemma 6.7.

Lemma 9. If for any $m \geqq 0$ and for any function $F_{1}(\delta)$ such that $F_{1}(\delta) \rightarrow \infty$ as $\delta \rightarrow 0$ one has

$$
\delta^{-1} \sum_{n=F_{1}(\delta)}^{\infty} \mu_{1}\left(U_{0, n, m}\right) \stackrel{\delta \rightarrow 0}{\longrightarrow} 0,
$$

then the inequality (12) holds. 
Let us assume now that the conditions of Lemma 9 fail, i.e. one can find $m_{1} \geqq 0$, $\varepsilon_{1} \geqq 0$ and a function $F_{1}(\delta) \stackrel{\delta \rightarrow 0}{\longrightarrow} \infty$ such that

$$
\sum_{n=F_{1}(\delta)}^{\infty} \mu_{1}\left(U_{0, n, m_{1}}\right) \geqq \varepsilon_{1} \delta .
$$

It follows from condition A that $\lambda_{n, 0}\left(T_{1}^{n} y\right) \rightarrow \infty$ as $n \rightarrow \infty$ for almost every point $y \in R_{-1}$ with respect to a metric in $R_{-1}$ that is induced by the metric in $M_{1}$. Hence for any such point $y \in R_{-1}$ one can find a neighborhood $V_{1}(y) \subset M_{1}$ such that $\lambda_{n, 0}\left(T_{1}^{n} y^{\prime}\right)>\Lambda^{m_{1}}$ for all $y^{\prime} \in V_{1}(y)$ if $n>n_{1}(y)$. Furthermore there exists smaller neighborhood $V_{2}(y) \subset V_{1}(y)$ such that $\lambda_{n, \delta}\left(T_{1}^{n} y^{\prime}\right)>\Lambda^{m_{1}}$ for all $n>n_{2}(y), y^{\prime} \in V_{2}(y)$, and $\delta<\delta_{2}(y)$. Thus according to the definition of $U_{0, n, m}$ the set $T_{1}^{n} U_{0, n, m_{1}}$ does not intersect $V_{2}(y)$ for any $\delta<\delta_{2}(y)$ and $n>n_{2}(y)$. Therefore for any $\varepsilon_{0}>0$ one can find numbers $\delta_{0}>0, n_{0}>0$ and a neighborhood $V_{0}(R) \subset M_{1}$ of the manifold $R$ such that

$$
\mu_{1}\left(V_{0}(R)\right) \cap\left(\bigcup_{n=n_{0}}^{\infty}\left(T_{1}^{n} U_{0, n, m_{1}}\right)\right)<\varepsilon_{0} \delta
$$

for all $\delta<\delta_{0}$. In view of the contradiction between (14) and (13) the relation (12) is proven.

Take now a point $y \in\{r(y)<\delta\} \backslash U_{0, \omega}$ and an integer $F(\delta)$. Consider the same smooth curve $\Sigma_{+, 0}^{(n)}$ as in the proof of Lemma 7, where $n>F(\delta)$. We put $\Sigma_{0, m}^{(n)}$ $=\widehat{T}_{m} \Sigma_{+, 0}^{(n)}$, where $0 \leqq m \leqq n$. Analogously to Lemma 7 one can prove (see [KSS2]),

Lemma 10. If a point $y \notin U_{0, \omega}$ then the length of the curve $\Sigma_{0, n-F(\delta)}^{(n)}$ is larger than $\delta \lambda_{F(\delta), \delta}^{-1}(y)$. Thus the curve $\Sigma_{0, n}^{(n)}$ intersects with the manifold of discontinuity of the transformation $T_{1}^{-n}$ for all $n$. Besides the distance in the $\ell_{1}$-metric induced on $\Sigma_{0, n}^{(n)}$ between the point $y$ and this manifold does not exceed $\delta$.

Recall (see Sect. 1) that the manifolds of discontinuity of the transformation $T_{1}^{k}$ consist for $k>0$ of the contracting and for $k<0$ of the expanding curves. In view of the condition G6 we get

Lemma 11. For any $\varepsilon_{1}>0$ there exists a neighborhood $U_{1}\left(x_{1}\right)$ and a number $\delta_{3}>0$ such that for $\delta<\delta_{3}$ a maximal angle between tangent vectors to $\Sigma_{0, n}^{(n)}, n>F(\delta)$, and tangent vectors to a corresponding manifold of discontinuity does not exceed $\varepsilon_{1}$.

The corollary to Lemma 11 is that the $\ell_{1}$-distance between $y$ and the corresponding manifold of discontinuity of $T_{1}^{F(\delta)}$ does not exceed $\varepsilon_{2} \delta$, where $\varepsilon_{2}=\varepsilon_{2}\left(\varepsilon_{1}\right)$ could be arbitrarily small if the neighborhood $U_{1}\left(x_{1}\right)$ was chosen sufficiently small. Hence the following statement holds.

Lemma 12. For any $\varepsilon_{2}>0$ there exists a neighborhood $U_{1}\left(x_{1}\right)$ such that the set $D_{\delta, \omega}=\{y: r(y)<\delta\} \backslash U_{0, \omega}$ belongs to the $\varepsilon_{2} \delta$-neighborhood of the manifold of discontinuity of the transformation $T_{1}^{F(\delta)}$ for all $\delta<\delta_{3}$.

We can construct the sets $U_{1}^{(\delta)}$ and $U_{0}^{(\delta)}$ that appeared in Lemma 2 and consist of "good" and "bad" parallelograms of the covering $\left\{G_{i}^{(\delta)}\right\}$ of $U_{1}\left(x_{1}\right)$ respectively.

Let the parallelogram $G_{i}^{(\delta)}$ intersect not more than one curve $\Sigma$ belonging to the manifold of discontinuity of the transformation $T_{1}^{ \pm F(\delta)}$. Suppose that $\varepsilon_{3}$ is a constant which is defined by a number $\varepsilon_{1}$ in condition a) of Lemma 2 and that this condition is valid for it. We put $G_{i}^{(\delta)} \subset U_{1}^{(\delta)}$ if $\mu_{1}\left(G_{i}^{(\delta)} \cap U_{1}^{(0)}\right) \geqq\left(1-\varepsilon_{3}\right) \mu_{1}\left(G_{i}^{(\delta)}\right)$. Also we 
take a neighborhood $U_{1}\left(x_{1}\right)$ and therefore $\varepsilon_{2}$ so small that the measure of the union of the $\varepsilon_{2} \delta$-neighborhoods of the boundary of $\partial G_{i}^{(\delta)}$ and of the set $\Sigma \cap G_{i}^{(\delta)}$ does not exceed $\frac{1}{2} \varepsilon_{3} \mu_{1}\left(G_{i}^{(\delta)}\right)$ for any $\delta$ and $G_{i}^{(\delta)}$.

Hence there exist two possibilities if $G_{i}^{(\delta)} \not \subset U_{1}^{(\delta)}$.

1. A parallelogram $G_{i}^{(\delta)}$ intersects not less than two curves of discontinuity of the transformation $T_{1}^{ \pm F(\delta)}$. In this case we put $G_{i}^{(\delta)} C U_{00}^{(\delta)}$.

2. $\mu_{1}\left(G_{i}^{(\delta)} \cap U_{0, \omega}\right)>\frac{1}{2} \varepsilon_{3} \mu_{1}\left(G_{i}^{(\delta)}\right)$. Then it follows from the property 3) of the coverings $\left\{G_{i}^{(\delta)}\right\}$ and from (10) that the measure of the union of such parallelograms does not exceed $\delta \phi_{5}(\delta)$, where $\phi_{5}(\delta) \rightarrow 0$ as $\delta \rightarrow 0$.

Thus Lemma 2 is proven and therefore Theorem 1 is proven as well.

We consider now the following important generalization of Theorem 1. Suppose that for a point $x$ one of the two following possibilities takes place:

1) $x \in M_{1}^{\prime} \backslash \mathscr{M}$ and the trajectory of $x$ hits one and only one curve of discontinuity $\Sigma_{k}$ of the transformation $T_{1}^{k}$ for some $k \neq 0$.

2) $x \in \Sigma_{k} \subset T_{1}^{k} V_{0}$ and its positive semitrajectory (if $k>0$ ) or its negative semitrajectory (if $k<0$ ) or both (if $k=0$ ) does not touch the boundary, does not contain singular points of $\partial Q$ and all its generalized semitrajectories do not reflect from $\partial Q^{0}$ only. We assume further that $x$ is an interior point of a smooth curve $\Sigma_{k}$. Then $\Sigma_{k}$ divides the neighborhood $U(x)$ into two parts $U_{1}$ and $U_{2}$. We could define the value $\Lambda_{n, 1}^{( \pm)}(x)\left(\Lambda_{n, 2}^{( \pm)}(x)\right)$ as the limit of $\Lambda_{n, 1}^{( \pm}(y)\left(\Lambda_{n, 2}^{( \pm)}(y)\right)$ as $y \rightarrow x$ and $y \in U_{1}\left(y \in U_{2}\right)$.

Theorem 1'. If a point $x_{1} \in M_{1}$ satisfies 1) or 2), where $k \geqq 0$ and $\Lambda_{n, i}^{(+)}\left(x_{1}\right) \rightarrow \infty$ as $n \rightarrow \infty$ (or $k \leqq 0$ and $\Lambda_{n, i}^{(-)}\left(x_{1}\right) \rightarrow \infty$ as $\left.n \rightarrow \infty\right), i=1,2$, then there exists a neighborhood of $x_{1}$ that belongs mod 0 to one and the same ergodic component.

Proof. It is easy to see that a sufficiently small neighborhood $U_{1}\left(x_{1}\right)$ does not intersect $\mathscr{M}$. Then it follows from Theorem 1 that each of the sets $U_{1}$ and $U_{2}$ belongs to one of the ergodic components. Furthermore in the proof of Theorem 1 it was shown that the set of points $y \in U_{1}\left(x_{1}\right)$ that has a LUM $W_{1}^{(u)}(y)$ which is shorter than $\delta$ can be divided into two subsets with the following properties. The measure of the first one equals $\delta \phi(\delta)$, where $\phi(\delta) \rightarrow 0$ as $\delta \rightarrow 0$ and the second belongs to a $\varepsilon \delta$-neighborhood of the manifold of discontinuity of the transformation $T_{1}^{-F(\delta)}$, where $F(\delta) \rightarrow \infty_{\text {s }}$ as $\delta \rightarrow 0$. Taking a sufficiently șmall neighborhood $U_{1}\left(x_{1}\right)$ we can choose $\varepsilon$ arbitrarily small.

Without any loss of generality we may suppose that $k \geqq 0$. For billiards under consideration the curves of discontinuity of the transformations $T_{1}^{k_{1}}$ and $T_{1}^{k_{2}}$ intersect transversally if $\operatorname{sgn} k_{1}=-\operatorname{sgn} k_{2}$ as was mentioned already in the previous section. Hence $\Sigma_{k}$ intersects transversally in $U_{1}\left(x_{1}\right)$ all manifolds $T_{1}^{s}\left(V_{0} \cup S_{0}\right)$, where $0<s \leqq n$. Therefore we can take a function $F(\delta)$ that tends to infinity as $\delta \rightarrow 0$ so slowly that the measure of the intersection of a $\delta$-neighborhood of the curve $\Sigma_{k}$ and a $\delta$-neighborhood of the manifolds of discontinuity of $T_{1}^{-F(\delta)}$ equals $\delta \phi_{6}(\delta)$, where $\phi_{6}(\delta) \rightarrow 0$ as $\delta \rightarrow 0$. So if $\delta$ is sufficiently small there is a set of positive measure that consists of LUMs with one endpoint belonging to $U_{1}$ and the other one belonging to $U_{2}{ }^{2}$ But this means that $U_{1} \cup U_{2}=U_{1}\left(x_{1}\right)$ belongs $\bmod 0$ to one ergodic component and Theorem $1^{\prime}$ is proven.

${ }^{2}$ If $x_{1} \in V_{0}$ then these LSMs consist of two smooth components 


\section{Ergodicity of a Class of Billiards with Focusing Components of the Boundary}

In this section we shall apply Theorem 1 to the proof of ergodicity of one concrete class of billiards. It was used to show ergodicity of semidispersing billiards in [SC, KSS1]. Our aim is the investigation of billiards in domains that have boundaries with at least one focusing component. We do not deal here with the most general case but instead consider a class of billiards for which verification of the conditions of Theorem 1 is rather visual. As usual to prove ergodicity one has to show that the set of points satisfying conditions of Theorem 1 or $1^{\prime}$ has measure one and is arcwise connected.

Let $Q$ be a domain in the Euclidean plane or on a two-dimensional torus with the Euclidean metric. We suppose that the boundary $\partial Q$ consists of a finite number of smooth (of class $C^{3}$ ) components that have curvature of constant sign or equal to zero. Besides assume that the following conditions are fulfilled:

(i) The boundary $\partial Q$ contains at least one focusing component.

(ii) Every focusing component $\Gamma^{F} \subset \partial Q$ has constant curvature, i.e. it is an arc of

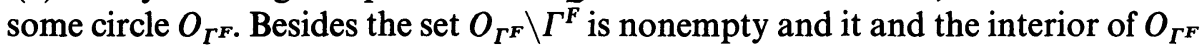
both belong to the interior of $Q$.

(iii) Dispersing components of the boundary $\partial Q$ intersect each other and neutral components of $\partial Q$ transversally.

(iv) The neutral part of the boundary $\partial Q^{0}$ consists of not more than two regular components if $Q \subset \mathbb{R}^{2}$ and not more than one if $Q \subset \mathbb{T}^{2}$. This condition means in particular that $\mu_{1}\left(\mathscr{M}^{+}\right)=\mu_{1}\left(\mathscr{M}^{-}\right)=0$.

The conditions (i)-(iii) were introduced in [B1]. Examples of domains satisfying them can be found in [B1-B3]. It is worthwhile to mention that these domains could have no neutral components in the boundary as well.

We have to stress that none of the conditions (i)-(iv) is necessary for ergodicity of the corresponding billiard. In particular focusing components of the boundary could have a nonconstant but slowly varying curvature [B4]. One can find in the papers $[\mathrm{W}, \mathrm{M}]$ some conditions on focusing components of $\partial Q$ when the corresponding billiards can have ergodic components of positive measure under some additional restrictions on the form of $Q$. Apparently (see [KMS, BKM]) the last condition (iv) is not needed. But this result of [KMS] is in the sense of category and cannot be applied directly to concrete polygons.

Theorem 2. $A$ billiard in a domain $Q$ that belongs to $\mathbb{R}^{2}$ or to a torus $\mathbb{T}^{2}$ with Euclidean metric and that satisfies conditions (i)-(iv) is ergodic.

Proof. We shall show that the set $\tilde{M}_{1} \subset M_{1}$ consisting of all points satisfying the conditions of Theorem 1 or of Theorem $1^{\prime}$ is arcwise connected and $\mu_{1}\left(\tilde{M}_{1}\right)=1$. Therefore any two points $x, y \in \tilde{M}_{1}$ one can join by a path that belongs to $\tilde{M}_{1}$. Due to its compactness this path could be covered by a finite number of neighborhoods such that according to Theorem 1 (or to Theorem $1^{\prime}$ ) every one of them belongs mod 0 to one ergodic component of the dynamic system generated by the automorphism $T_{1}$. Hence the union of these neighborhoods belongs mod 0 to one ergodic component. Therefore the set $\tilde{M}_{1}$ belongs mod0 to one ergodic component. In view of the equality $\mu_{1}\left(\tilde{M}_{1}\right)=1$ we get the ergodicity of $T_{1}$. 
Let $m_{-}(x)=\min _{m>0}\left\{m: \pi\left(T_{1}^{-m} x\right) \in \partial Q^{+} \cup \partial Q^{-}\right\}, m_{+}(x)=\min _{m>0}\left\{m: \pi\left(T_{1}^{m} x\right) \in \partial Q^{+}\right.$ $\left.\cup \partial Q^{-}\right\}$. If $x \in M_{1}^{\prime} \backslash \mathscr{M}^{-}\left(x \in M_{1}^{\prime} \backslash \mathscr{M}^{+}\right)$then $m_{-}(x)<\infty\left(m_{+}(x)<\infty\right)$. In view of (iv) there exists $m_{0}$ such that $m_{-}(x)<m_{0}, m_{+}(x)<m_{0}$ for any $x \in M_{1}$, if neutral components of $\partial Q$ are nonparallel. Anyway $M_{1} \backslash \mathscr{M}$ is arcwise connected.

We take an arbitrary point $x_{0} \in \pi^{-1}\left(\Gamma^{F}\right) \subset \pi^{-1}\left(\partial Q^{-}\right)$and denote by $p_{ \pm}\left(x_{0}\right)$ a minimal positive integer such that $\pi\left(T_{1}^{p+\left(x_{0}\right)} x_{0}\right) \notin \Gamma^{F}$ and correspondingly $\pi\left(T_{1}^{-p_{-}\left(x_{0}\right)} x_{0}\right) \notin \Gamma^{F}$. Consider $N=\left\{x \in M_{1}^{\prime}: \pi(x) \in \partial Q^{-}, p_{+}(x)=\infty\right.$ or $\left.p_{-}(x)=\infty\right\}$. It is clear that $N$ is the union of all periodic trajectories of a billiard that have reflections with one and the same focusing component of the boundary. Such trajectories exist if the focusing component $\Gamma^{F}$ under consideration is larger than a semicircle.

Recall that in coordinates $(r, \phi)$ the phase space $M_{1}$ of the billiard is a cylinder or a union of a finite number of cylinders.

Lemma 13. The subset $N$ consists of a finite number of segments of horizontal curves that have form $\{\phi=$ const $\}$ in $M_{1}$. Besides the complement $M^{1} \backslash N$ is arcwise connected.

Proof. It follows from (ii) that only segments $\{\phi=$ const $\}$ belong to $N$ which corresponds to a family of periodic trajectories of our billiard that have reflections with one and the same focusing component of $\partial Q$.

Since $\partial Q \neq \Gamma^{F}$ and due to (ii) the set $M_{1} \backslash N$ is arcwise connected.

Let the divergent beam of trajectories have a series of consecutive reflections from the dispersing part of the boundary. Then to such a series corresponds a segment of a continued fraction (7) with positive elements. It is known that if a continued fraction with positive elements is convergent then the sequences of convergents with odd and with even indices tend to the same limit from above and from below correspondingly. This property allows one to construct families of invariant cones for Sinai billiards [S1].

For billiards with focussing components the situation is more delicate. In this case the odd elements of the continued fraction (7) have the same sign and the even elements have the opposite sign. It was shown in [B1, B3] that for the billiards under consideration the sequence of all convergents of (7) is a monotonic one. The crucial role for these billiards is played by the conditions of defocussing G4-G5. We shall discuss now the main property that makes it possible to construct families of invariant cones for the billiards under consideration.

Suppose that an infinitesimal divergent beam of trajectories is incident on the focusing component $\Gamma^{F} C \partial Q$ with curvature $k^{(0)}$ and suffers a series of $n$ subsequent reflections from $\Gamma^{F}$. Denote by $\tau$ the interval of time between any two consecutive reflections from this series, and by $\phi$ the angle of incidence that is constant for such series.

The following lemma was proved in [B 1] (as Assertion 1). We shall denote by $\kappa_{-}^{(0)}$ the curvature of a beam of trajectories at the moment just before a reflection from the boundary $\partial Q$.

Lemma 14. Let the curvature $\kappa_{-}^{(0)}$ of a divergent infinitesimal beam of trajectories that is incident on $\Gamma^{F}$ satisfy the inequality $0<\kappa_{-}^{(0)}<\frac{2}{\tau}$. Then a corresponding series 
of consecutive reflections from $\Gamma^{F}$ of this beam satisfies the following conditions: (F1) At a moment just after any reflection from $\Gamma^{F}$ the considered beam is convergent and its curvature $\kappa_{+}^{(k)}$ at this moment satisfies the inequality $\kappa_{+}^{(k)}<-2 / \tau$, where $1 \leqq k \leqq n$.

(F2) For any $k, 1 \leqq k \leqq n-1$, the beam under consideration goes through a conjugate point and is incident on $\Gamma^{F}$ as a divergent one, i.e. $\kappa_{-}^{(k)}>0$.

(F3) $\left|\kappa_{+}^{(k)}\right|>\kappa_{-}^{(k)}$.

Lemma 14 shows that the class of billiards under consideration is similar to Sinai billiards. Indeed it says that between any two consecutive reflections from one and the same focusing components of the boundary each expanding beam increases its $\ell_{1}$-length. In Sinai billiards this property holds with respect to the flow $\left\{S^{t}\right\}$ as well but in our billiards it is valid with respect to $\left\{T^{n}\right\}$ only.

Thus we can use the following ideas to construct families of invariant cones in this case. From (i)-(iv) and Lemma 14 we have that if an infinitesimal beam of trajectories was plane (neutral) before the first reflection from $\partial Q^{+} \cup \partial Q^{-}$then it would be divergent before each of its consecutive reflections from the same component of $\partial Q$. Further, after each reflection from $\partial Q^{+} \cup \partial Q^{0}\left(\partial Q^{-}\right)$it would be divergent (convergent). In addition if the reflection takes place in $\partial Q^{-}$then its path up to the conjugate point is less than half of the total path up to the next reflection from $\partial Q$.

The families of invariant cones are given via the following relations for billiards satisfying (i)-(iv):

( 1 1) $\pi(x) \in \partial Q^{+}, \pi\left(T_{1}^{-m_{-}(x)} x\right) \in \partial Q^{+}$.

$(\alpha 2) \pi(x) \in \partial Q^{+}, \pi\left(T_{1}^{-m_{-}(x)} x\right) \in \partial Q^{-}$.

$$
K_{1}^{(+)}(x)=k^{(0)}(r) \leqq \frac{d \phi}{d r} \leqq k^{(0)}(r)+\frac{\cos \phi(x)}{\sum_{k=1}^{m-(x)} \tau\left(T_{1}^{-k} x\right)}=K_{2}^{(+)}(x)
$$

(๔3) $\pi(x) \in \partial Q^{(0)}, \pi\left(T_{1}^{-m_{-}(x)} x\right) \in \partial Q^{+}$.

$$
K_{1}^{(-)}(x)=k^{(0)}(r) \leqq \frac{d \phi}{d r} \leqq k^{(0)}(r)+\frac{2 \cos \phi(x)}{\sum_{k=1}^{m-(x)} \tau\left(T_{1}^{-k} x\right)}=K_{2}^{(-)}(x) .
$$

$$
\begin{aligned}
& \frac{k^{(0)}\left(\pi\left(T_{1}^{-m_{-}(x)} x\right)\right)}{1+\sum_{k=1}^{m-(x)} \tau\left(T_{1}^{-k} x\right) k^{(0)}\left(\pi\left(T_{1}^{-m_{-}(x)} x\right)\right)} \\
& \leqq \frac{d \phi}{d r} \leqq \frac{\cos \phi\left(T_{1}^{-m_{-}(x)} x\right)}{1+K_{2}^{(0)}\left(\pi\left(T_{1}^{-m_{-}(x)} x\right)\right)+\frac{\left.T_{1}^{-m_{-}(x)} x\right) \sum_{k=1}^{m-(x)+m_{-}(x)+1} \tau\left(T_{1}^{-k} x\right)}{\left.m_{-1}^{-m_{-}(x)} x\right)} \tau\left(T_{1}^{-k} x\right)}
\end{aligned}
$$

$(\alpha 4) \pi(x) \in \partial Q^{(0)}, \pi\left(T_{1}^{-m_{-}(x)} x\right) \in \partial Q^{-}$.

$$
\frac{K_{1}^{(-)}\left(T_{1}^{-m_{-}(x)} x\right)}{1+K_{1}^{(-)}\left(T_{1}^{-m_{-}(x)} x\right) \sum_{k=1}^{m-(x)} \tau\left(T_{1}^{-k} x\right)} \leqq \frac{d \phi}{d r} \leqq \frac{K_{2}^{(-)}\left(T_{1}^{-m_{-}(x)} x\right)}{1+K_{2}^{(-)}\left(T_{1}^{-m_{-}(x)} x\right) \sum_{k=1}^{m-(x)} \tau\left(T_{1}^{-k} x\right)} .
$$


We define contracting cones $K^{(s)}(x)$ in the following way:

(ß1) $\pi(x) \in \partial Q^{+}, \pi\left(T_{1}^{m_{+}(x)} x\right) \in \partial Q^{+}$.

$$
\bar{K}_{1}^{(+)}(x)=-k^{(0)}(r)-\frac{\cos \phi(x)}{\sum_{k=1}^{m+(x)-1} \tau\left(T_{1}^{k} x\right)} \leqq \frac{d \phi}{d r} \leqq-k^{(0)}(r)=\bar{K}_{2}^{(+)}(x) .
$$

(ß2) $\pi(x) \in \partial Q^{+}, \pi\left(T_{1}^{m_{+}(x)} x\right) \in \partial Q^{-}$.

$$
\bar{K}_{1}^{(-)}(x)=-k^{(0)}(r)-\frac{2 \cos \phi}{\sum_{k=1}^{m+(x)-1} \tau\left(T_{1}^{k} x\right)} \leqq \frac{d \phi}{d r} \leqq-k^{(0)}(r)=\bar{K}_{2}^{(-)}(x) .
$$

(B3) $\pi(x) \in \partial Q^{(0)}, \pi\left(T_{1}^{m+(x)} x\right) \in \partial Q^{+}$.

$$
\frac{\bar{K}_{1}^{(+)}\left(T_{1}^{m+(x)} x\right)}{1+\bar{K}_{1}^{(+)}\left(T_{1}^{m_{+}(x)} x\right) \sum_{k=1}^{m+(x)-1} \tau\left(T_{1}^{k} x\right)} \leqq \frac{d \phi}{d r} \leqq \frac{\bar{K}_{2}^{(+)}\left(T_{1}^{m+(x)} x\right)}{1+\bar{K}_{2}^{(+)}\left(T_{1}^{m_{+}(x)} x\right) \sum_{k=1}^{m+(x)-1} \tau\left(T_{1}^{k} x\right)}
$$

(ß4) $\pi(x) \in \partial Q^{(0)}, \pi\left(T_{1}^{m+(x)}\right) \in \partial Q^{-}$.

$$
\frac{\bar{K}_{1}^{(-)}\left(T_{1}^{m+(x)} x\right)}{1+\bar{K}_{1}^{(-)}\left(T_{1}^{m_{+}(x)} x\right) \sum_{k=1}^{m+(x)-1} \tau\left(T_{1}^{k} x\right)} \leqq \frac{d \phi}{d r} \leqq \frac{\bar{K}_{2}^{(-)}\left(T_{1}^{m_{+}(x)} x\right)}{1+\bar{K}_{2}^{(-)}\left(T_{1}^{m_{+}(x)} x\right) \sum_{k=1}^{m+(x)-1} \tau\left(T_{1}^{k} x\right)}
$$

Making use of $(1-4),\left(3^{\prime}\right),\left(4^{\prime}\right)$ it is easy to check (see also [B1]) that the constructed systems of cones both satisfy the condition G1.

Furthermore according to the definition, the set $R_{0}$ consists of smooth components that are given by the equations $\frac{d \phi}{d r}=0$ and $\frac{d \phi}{d r}=\infty$. By direct calculation using (1), (2), (4), (4') (see Lemma 3 in [B1] or Lemma 3 in [B3] for details) one can show that each smooth component of the set $T_{1} R_{0}\left(T_{1}^{-1} R_{0}\right)$ is an expanding (contracting) curve. Hence $\mathrm{G} 2$ follows in view of the invariance of the constructed systems of cones.

The property of hyperbolicity G3 was proved for the class of billiards under consideration in [B1] (see also [W]).

It follows from (ii) and from Lemma 14 that both the properties of defocussing (G4) and of strong defocussing (G5) are fulfilled for our class of billiards. So according to Proposition 3 the constructed cones are perfect ones.

The property $\mathrm{G} 6$ for these billiards was proven in [B1] (Lemmas 6-8, see also Sect. 2, 3 in [B3]).

One has to mention that our cones are constructed at all points of $M_{1} \backslash(N \cup \overline{\mathscr{M}})$, where $T_{1}^{-m_{-(x)}} x\left(T_{1}^{m+(x)} x\right)$ is defined.

We check now condition $A$. It is clear that both the sets $R_{1} \cap N$ and $R_{-1} \cap N$ contain at most a finite number of points. Hence in view of (i)-(iv), $(\alpha 1)-(\alpha 4)$, and $(\beta 1)-(\beta 4) A$ is fullfilled for all points $x \in R_{1}\left(x \in R_{-1}\right)$ such that a positive (negative) semitrajectory of $x$ does not touch the boundary, does not hit singular points of $\partial Q$ and does not reflect solely from the neutral part of $\partial Q$. Denote the set that consists of all such points by $F$. 
Recall (see Sect. 1) that sets $T_{1}^{k} S_{0}$ and $T_{1}^{k} V_{0}$ consist of expanding (contracting) curves if $k>0(k<0)$. Further, $V_{0}$ consists of vertical segments $r=\hat{r}_{i}, i=1, \ldots, L$, and $S_{0}$ consists of horizontal segments $\phi= \pm \pi / 2$. Every set $\bigcup_{k=-\infty}^{\infty} T_{1}^{k} V_{0}$ and $\bigcup_{k=-\infty}^{\infty} T_{1}^{k} S_{0}$ contain no more than a countable number of corresponding curves and segments. Therefore the set $F$ has full measure in $R_{1}\left(R_{-1}\right)$ with respect to the measure induced in this set by $\mu$.

Furthermore the set that consists of all points of intersection of at least two regular components of the $\operatorname{set}\left(\bigcup_{k=-\infty}^{\infty} T_{1}^{k} S_{0} \cup \bigcup_{k=-\infty}^{\infty} T_{1}^{k} V_{0}\right)$ has codimension two. Hence its complement is arcwise connected.

Therefore for billiards that satisfy (i)-(iv) all the conditions of Theorems 1 and $1^{\prime}$ are fulfilled for an arcwise connected subset $\tilde{M}_{1} \subset M_{1}$, where $\mu_{1}\left(\tilde{M}_{1}\right)=1$.

Thus the transformation $T_{1}$ and hence the flow $\left\{T^{t}\right\}$ also are ergodic. It follows from the general theory of billiards and of hyperbolic dynamic systems with singularities (see [KS], [GO]) that billiards of the class under consideration are Bernoullian dynamical systems as well.

\section{Concluding Remarks}

As was mentioned in the introduction the main theorem of the theory of billiards with hyperbolic behavior was first formulated and proved by Sinai in [S1]. He suggested also both methods existing now to prove it; they will be referred to in the following as the old (see [S1, BS]) and the new (see [S2, S3, SC, KSS1, KSS2]) method.

The formulation of this theorem did not change essentially passing from the old method of proof of the new one. In both cases it states that, for a generic point $x$ of the phase space of billiards belonging to some peculiar class of billiards with hyperbolic behavior, there exists a neighborhood $U(x)$ that belongs mod 0 to one ergodic component. The crucial element of the proof is to show that if $U(x)$ is sufficiently small and if a parallelogram $G$ is contained in $U(x)$ then the set consisting of all points $y \in G$ such that the LUM $W_{1}^{(u)}(y)$ and the LSM $W_{1}^{(s)}(y)$ intersect both sides of $G$ with the same monotonicity as $W_{1}^{(s)}(y)$ and $W_{1}^{(u)}(y)$ respectively has close to 1 (conditional) measure in $G$. So the idea of the main theorem is to demonstrate that in the phase space of billiards from the class under consideration the process of expansion of expanding curves generated by hyperbolicity is more effective than the process of their fractioning caused by hitting of singularities and tangency with the boundary.

The old method of proof of the main theorem deals with the detailed investigation of the fine structure of the manifolds of singularity of $T_{1}$ and of their neighborhoods. The advantage of the new method is that it deals with more general properties of these neighborhoods. It is worthwhile to mention that the old method allows one to make quite visible the analogy between structures of singular manifolds in Sinai billiards and in billiards with focusing components of the boundary and hyperbolic behavior.

The new method of proof of the main theorem is much simpler than the old one. [Nevertheless one has to mention that the assertion of the main theorem in its old 
version is stronger (at least formally) than in the new one.] In forthcoming papers we shall demonstrate that it could be applied to the proof of ergodicity of more general classes of billiards, in particular many-dimensional ones.

Acknowledgements. I am very indebted to Ya. G. Sinai and Ya. B. Pesin for useful discussions. I want to express my sincere gratitude to N. I. Chernov, who had read the whole manuscript and made many important suggestions and useful remarks. I also want to thank G. Bolz and T. Krüger for their help in preparing the manuscript.

\section{References}

[BKM] Boldrighini, C., Keane, M., Marchetti, F.: Billiards in polygons. Ann. Probab. 6, 532-540 (1978)

[B1] Bunimovich, L.A.: On billiards closed to dispersing. Matem. Sbornik 95, 49-73 (1974)

[B 2] Bunimovich, L.A.: On ergodic properties of some billiards. Funk. Anal. Appls. 8, 73-74 (1974)

[B 3] Bunimovich, L.A.: On the ergodic properties of nowhere dispersing billiards. Commun. Math. Phys. 65, 295-312 (1979)

[B4] Bunimovich, L.A.: On the stochastic dynamics of rays in resonators. Radiofizika 28, 1601-1602 (1985)

[BS] Bunimovich, L.A., Sinai, Ya.G.: On the main theorem of the ergodic theory of dispersing billiards. Mat. Sbor. 90, 415-431 (1973)

[CFS] Cornfeld, I.P., Fomin, S.V., Sinai, Ya.G.: Ergodic theory, p. 383. Moscow: Nauka 1980

[GO] Gallavotti, G., Ornstein, D.: Billiards and Bernoulli schemes. Commun. Math. Phys. 38, 83-101 (1974)

[H] Hopf, E.: Statistik der geodätischen Linien in Mannigfaltigkeiten negativer Krümmung. Ber. Verch. Akad. Wiss. Leipzig 91, 261-304 (1939)

[KS] Katok, A., Strelcyn, J.-M.: Smooth maps with singularities. Lecture Notes in Mathematics, Vol. 1222, pp. 283. Berlin, Heidelberg, New York: Springer 1986

[KMS] Kerckhoff, F., Masur, H., Smillie, J.: Ergodicity of billiard flows and quadratic differentials. Ann. Math. 124, 293-311 (1986)

[KSS1] Krámli, A., Simányi, N., Szász, D.: Three billiard balls on the $v$-dimensional torus is a $K$-flow, Preprint No. 38. Math. Inst. of Hung. Ac. Sci., 1988, 40pp

[KSS2] Krámli, A., Simányi, N., Szász, D.: "Transversal" fundamental theorem for semidispersing billiards. Commun. Math. Phys. (in press)

[M] Markarian, R.: Billiards with Pesin region of measure one. Commun. Math. Phys. 118, 87-97 (1988)

[S 1] Sinai, Ya.G.: Dynamical systems with elastic reflections. Sov. Math. Surv. 5, 141-192 (1970)

[S2] Sinai, Ya.G.: Ergodic properties of the Lorentz gas. Funk. Anal. Appl. 13, 46-59 (1979)

[S3] Sinai, Ya.G.: Development of Krylov ideas. An addendum to the book: N.S. Krylov. Works on the foundation of statistical physics, pp. 239-281. Princeton, NJ: Princeton University Press 1979

[SC] Sinai, Ya.G., Chernov, N.I.: Ergodic properties of some systems of two-dimensional disks and of three-dimensional balls. Sov. Math. Surv. 42, 153-174 (1987)

[W] Wojtkowski, M.: Principles for the design of billiards with nonvanishing Lyapunov exponents. Commun. Math. Phys. 105, 391-414 (1986) 
